\title{
Generalizing Ellipsoidal Growth
}

Gabriella Maria Silveira de Sáa ${ }^{\circledR}$, Mariana Sizenando Lyrio ${ }^{a}$, André Luiz Moraes Alves ${ }^{a}$,

Harison da Silva Ventura ${ }^{a}$, Weslley Luiz da Silva Assis ${ }^{a}$, Elena Villa ${ }^{b}$, Paulo Rangel Rios ${ }^{a}$

\author{
${ }^{a}$ Escola de Engenharia Industrial Metalúrgica de Volta Redonda, Universidade Federal Fluminense, Av. \\ dos Trabalhadores, 420, CEP 27.255-125, Volta Redonda, RJ, Brasil \\ ${ }^{b}$ Dipartimento di Matematica, Università degli Studi di Milano, via Saldini 50, 20133, Milano, Italia
}

Received: March 13, 2019; Revised: April 24, 2019; Accepted: April 29, 2019

\begin{abstract}
This paper generalizes previous work of Rios and Villa on spherical growth. The generalized equation applies to nucleation of ellipsoids according to an inhomogeneous Poisson point process. Microstructural evolution in three dimensions of nucleation and growth transformations of ellipsoids is simulated using the causal cone method. In the simulation, nuclei are located in space according to an inhomogeneous Poisson point process. The transformed regions grow with prolate and oblate ellipsoidal shapes. The ellipsoids have their corresponding axes parallel. The simulation and the exact analytical solution are in excellent agreement. Microstructures generated by the computer simulation are displayed. From these generated microstructures one can obtain the contiguity. In the contiguity against volume fraction plot, data from the sphere and all ellipsoids fall on the same curve. The contiguity curve for nucleation according to an inhomogeneous Poisson point process falls above the contiguity curve for nucleation according to a homogeneous Poisson point process. This behavior indicates that nucleation according to an inhomogeneous Poisson point process introduced a nucleus clustering effect.
\end{abstract}

Keywords: Microstructure, analytical methods, phase transformations, recrystallization, ellipsoidal growth.

\section{Introduction}

The grains of polycrystals are usually equiaxed. In other words, the microstructure is isotropic. Nonetheless, because of processing, the grain shape may be elongated and the microstructure anisotropic.

For example, when a polycrystal is deformed by rolling, cold-drawing or ECAP ${ }^{1}$ the microstructure becomes anisotropic. Another example is the formation of ferrite from heavily deformed austenite ${ }^{2}$. In this situation, the ferrite grains may form elongated instead of equiaxed ${ }^{2}$. A less common example is the precipitation in a Ti-20wt\%Mo alloy under compressive stress $^{3}$ that results in parallel ellipsoidal precipitates. Ellipsoids are the first approximation for elongated grains. Therefore, it is of interest to study the formation of ellipsoidal grains in detail. In this paper, we use computer simulation and analytical methods to carry out this study.

One typically associates the classic work of JohnsonMehl, Avrami, and Kolmogorov (JMAK) ${ }^{4-6}$ with the growth of spherical grains. Nonetheless, Kolmogorov in his early work already stated that ${ }^{5}$ : "The formulas hold either under the assumption of uniform growth in all directions (spherical growth) or for regions of arbitrary shape similarly oriented in space" (emphasis is ours).

This excerpt means that Kolmogorov already knew that his result could be valid for ellipsoidal growth provided the corresponding axes where parallel. Considering site

*e-mail: gabriellasilveira@id.uff.br saturation nucleation, that is, new regions nucleate at $t=0$ and grow with an ellipsoidal shape with a constant rate the expression for ellipsoidal growth is

$$
V_{V}(t)=1-\exp \left(-\frac{4 \pi}{3} \lambda G_{1} G_{2} G_{3} t^{3}\right)
$$

In Eq. (1) the growing regions keep their shape during growth. $G_{1}, G_{2}$ and $G_{3}$ are the growth velocities along the principal axes and $\lambda$ is the number of nuclei per unit of volume. The axes lengths are $a_{1}=G_{1} t, a_{2}=G_{2} t, a_{3}=G_{3} t$. Clearly if $G_{1}=G_{2}=G_{3}$ then Eq. (1) reduces to the usual expression for spherical growth.

The critical issue here is Kolmogorov's assertive that regions must be similarly oriented in space. Eq. (1) is not valid if the ellipsoids are randomly oriented in space. Eq. (1) is not valid because if one has ellipsoids randomly oriented during a nucleation and growth transformation, one ellipsoid may prevent the growth of another. This phenomenon is called blocking or shielding ${ }^{7,8}$.

There have been many studies on this problem, and approximate solutions were proposed ${ }^{7,8}$. Still, an exact mathematical solution to this problem is unavailable. Godiksen et al. ${ }^{9}$ performed computer simulation of uniform randomly oriented oblate and prolate ellipsoids. Godiksen et al.'s results showed that the particular forms of Eq. (1) for prolates and oblates spheroids given by Vandermeer et al. ${ }^{10}$ are valid if 
the ratio of the largest to the smallest ellipsoidal axis is less than four. For example, prolate ellipsoids with axes 4:1:1 still obeyed Eq. (1) but not with axes 8:1:19.

In all those cases the nucleation of the ellipsoids was supposed to originate on sites uniform randomly located in space. In other words, nucleation sites that are located according to a homogeneous Poisson point process ${ }^{11}$. In addition to these nucleation and growth studies, exciting work has been done on the influence of anisotropy in the final nonequilibrium grain size distribution ${ }^{12,13}$.

In previous work, Rios and Villa ${ }^{14}$ generalized JMAK's treatment so that nuclei can be located in space not only according to a homogeneous Poisson point process but also according to an inhomogeneous Poisson point process. As a result, instead of volume fraction one now has a position-dependent mean volume density, $V_{v}(t, x)$. Rios and Villa still managed to give a simple expression for the generalized site saturated case with constant and isotropic velocity, $G$, that is spherical growth

$$
V_{V}(t, x)=1-\exp \left(-\frac{4 \pi}{3} \lambda(x) G^{3} t^{3}\right)
$$

where $\lambda(x)$ is the position dependent intensity of an inhomogeneous Poisson point process.

One readily recognizes that when one has a homogeneous Poisson point process, $\lambda$ is independent of the position and is equal to the number of nuclei per unit of volume. If $\lambda$ is a constant, Eq. (2) reduces to the well-known JMAK's formula.

Nevertheless, for Eq. (2) to have such a convenient expression Rios and Villa assumed that $\lambda(x)$ was a harmonic function, that is, $\Delta \lambda(x)=0$. Otherwise, if $\lambda(x)$ were not harmonic, Rios and Villa showed that one still could obtain an expression for an arbitrary inhomogeneous Poisson point process, but it would involve integrals, see ref. ${ }^{14}$ for details. Eq.(2) was tested against computer simulation using cellular automata in a previous work ${ }^{15}$.

Because of this, one cannot merely introduce $\lambda(x)$ into Eq. (1) "by analogy" to obtain an expression for ellipsoids nucleated according to an inhomogeneous Poisson point process.

In this paper, we generalize Eq. (1). The nucleation of ellipsoids follows an inhomogeneous Poisson point process. We determine the conditions that $\lambda(x)$ should satisfy so that one can still have a convenient expression such as Eq. (2). We test this expression against computer simulation of the nucleation and growth of prolate and oblate ellipsoids similarly oriented in space for several ratios of the axis's length.

\section{Mathematical Background}

As demonstrated by Rios and Villa ${ }^{14}$, the critical point for obtaining Eq. (2) in such a convenient form is a property of integrals of harmonic functions: the mean value property. In $3 \mathrm{D}$, this property states that the integral of a harmonic function $f$ within a ball of unit ratio centered at $x$ is equal to $(4 \pi / 3) f(x)$. For details see Rios and Villa ${ }^{14}$. In what follows we derive which conditions should the function $f$ satisfy so that a similar property applies. This similar property is that, instead of a ball centered at the origin, one has an ellipsoid centered at the origin. From this property, an expression like Eq. (2) can be obtained for ellipsoids.

For the sake of simplicity, let us consider the case of site saturation. Site-saturation means that all nucleation sites are exhausted early in the transformation. In other words, all nucleation takes place a $t=0$, and no more nucleation takes place during further transformation progress.

Let the growth model be as follows: the grains grow with an ellipsoidal shape with a constant rate. That is, at time $t$ the grain born at point $x$ is given by an ellipsoid centred at $x$ with axes of length $a_{1}, a_{2}$ and $a_{3}$, respectively. The ellipsoids are oriented in space so that their corresponding axes are parallel. Thus, let us denote by $E\left(x ; a_{1}, a_{2}, a_{3}\right)$ the ellipsoid in $\mathbb{R}^{3}$ centered in $x=\left(x_{1}, x_{2}, x_{3}\right)$ with axes $a_{1}, a_{2}$ and $a_{3}$, and such that the direction of the $a_{1}$-axis is along the vector $(1,0,0)$.

$$
\begin{aligned}
& E\left(X ; a_{1}, a_{2}, a_{3}\right)=\left\{\left(y_{1}, y_{2}, y_{3}\right) \in \mathbb{R}^{3}: \frac{\left(y_{1}-x_{1}\right)^{2}}{a_{1}^{2}}+\right. \\
& \left.\frac{\left(y_{2}-x_{2}\right)^{2}}{a_{2}^{2}}+\frac{\left(y_{3}-x_{3}\right)}{a_{3}^{2}} \leq 1\right\}
\end{aligned}
$$

In this way we can model the nucleation process by a point process $N=\left\{x_{n}\right\}$ in $\mathbb{R}^{3}$ where $x_{n}$ represents the spatial location of the $n$-th nucleus of the ellipsoidal grain. Thus, denoted by $\theta^{t}(x)$ the grain born at point $x$ and grown until time $t$, we have that the crystallized region $\theta^{t}$ at time $t$ is the random closed set

$$
\Theta^{t}:=\bigcup_{\left(x_{n}\right) \in N} \Theta^{t}\left(x_{n}\right)
$$

where

$$
\Theta^{t}\left(x_{n}\right)=E\left(x_{n} ; a_{1}, a_{2}, a_{3}\right)
$$

The causal cone $C(t, x)$ at time t at point $x$ is then given by

$$
C(t, x)=\left\{\mathrm{y} \in \mathbb{R}^{3}: \mathrm{y} \in \Theta^{t}(x)\right\}
$$

It follows that

$$
\Lambda(C(t, x))=\int_{\Theta^{\prime}(x)} \lambda(\mathrm{y}) \mathrm{dy}
$$

As we well know ${ }^{14}$, if the nucleation process $N$ is a Poisson point process, then the mean volume density $V_{v}(t, x)$ is given by

$$
V_{V}(t, x)=1-e^{-\Lambda(C(t, x))}
$$

The problem is now to compute the integral in Eq. (7). 
If the intensity $\lambda(y)$ is a function in $\mathbb{R}^{3}$ such that

$\sum_{i=1}^{3} \partial_{i}^{2} f(\mathrm{y})=0$

where $f(y)=\lambda\left(a_{1} y_{1}+x_{1}, a_{2} y_{2}+x_{2}, a_{3} y_{3}+x_{3}\right)$.

Eq. (9) ensures that has the mean value property for ellipsoids. Therefore, following a similar procedure described in Rios and Vila ${ }^{14}$ one can integrate Eq. (7)

$V_{V}(t, x)=1-\exp \left\{-\lambda(x) \frac{4}{3} \pi G_{1} G_{2} G_{3} t^{3}\right\}$

Note that, the case of spherical growth is a particular case of the ellipsoidal case where $G_{1}=G_{2}=G_{3}$.

In the above one obtained an expression for a spatially dependent but time-independent number of nuclei. Following the same reasoning as above $\mathrm{e}^{14}$, we can also derive an expression for a spatially dependent but time-dependent number of nuclei: a constant nucleation rate, $I(x)$, i. e., a constant (but spatially dependent) nucleation rate

$V_{V}(t, x)=1-\exp \left\{-I(x) \frac{\pi}{3} G_{1} G_{2} G_{3} t^{4}\right\}$

The function, $I(x)$, must satisfy the same criterion derived for $\lambda(x)$ expressed by Eq. (9).

Regarding a constant nucleation rate in the context of an inhomogeneous Poisson point process, Eq. (11), refer to Rios and Villa ${ }^{14}$.

Returning to Eq. (10) one may notice that an intensity function $\lambda$ of the type

$$
\lambda(x)=a x_{1}+b
$$

satisfies Eq. (9).

Moreover, one may also define a normalized time for each transformation as $\tau=\sqrt[3]{G_{1} G_{2} G_{3}} t$, Eq. (2) and Eq. (10) can be rewritten as Eq. (13). Note that, in this case, each transformation has its normalized time that depends on the geometric parameters $a_{1}, a_{2}$ and $a_{3}$

$$
V_{V}(t, x)=1-\exp \left\{-\lambda(x) \frac{4}{3} \pi \tau^{3}\right\}
$$

The normalized time will be useful in what follows as we will be able to combine in the same plot the kinetics of ellipsoids of different shapes.

The volume fraction transformed can be defined as the integral of the mean volume density over the whole volume $[0,1] \times[0,1] \times[0,1]$

$$
\mathbf{V}_{\mathbf{v}}(t)=\iiint_{0}^{1} V_{V}(t, x) d x
$$

\section{Methodology}

For the nucleation and growth process, one performed a computer simulation using the Causal Cone method ${ }^{16}$.
The matrix comprised $300 \times 300 \times 300$ cubic cells. Prolate ellipsoids with aspect ratios 8:1:1, 4:1:1 and 2:1:1 and oblate ellipsoids with aspect ratios $8: 8: 1,4: 4: 1$ and 2:2:1 were simulated. Simulations employing spherical growth were also carried out for comparison. All simulations used site-saturated nucleation described above.

The nuclei were located within the matrix according to an inhomogeneous Poisson point process with an intensity, $\lambda(x)$, varying along only one direction $x_{1}$. The intensity was equal to $\lambda(x)=\lambda\left(x_{1}, x_{2}, x_{3}\right)=m x_{1}+$ $n$ where " $m$ " and " $n$ " are constants equal to 596 and 2, respectively. The same values of " $m$ " and " $n$ " were used in an earlier work of in which growth was equiaxed ${ }^{15}$. For dimensional purposes, we consider that each the side of a cell measures $1 \mu \mathrm{m}$. Therefore, the matrix has a volume equal to $300^{3} \mu \mathrm{m}^{3}$. These numbers were chosen to give a total number of nuclei equal to 300 . As a result, the mean grain size is roughly $44 \mu \mathrm{m}$ that is a reasonable value. Periodic boundary conditions were adopted except along the $x_{1}$ axis. All growth velocities were kept constant throughout the transformation. Each quantity reported here is the mean value of 50 simulations. That is, we repeated each simulation 50 times. This number of repetitions, 50, was used in several of our previous work with reliable results. The function $\lambda(x)$ used here was similar to that used in a previous work in which the regions were equiaxed ${ }^{15}$.

\section{Results and Discussion}

\subsection{Microstructural evolution}

Figure 1 shows the microstructural evolution for oblate ellipsoidal grains with aspect ratios $4: 4: 1$. There is an increase in the mean volume density, $V_{V}(t, x)$, from $x_{1}=0$ to $x_{1}=1$. This is consistent with the fact that nuclei density increases with $x_{1}$.

Therefore, the lower part of the matrix contains larger grains as it has fewer nuclei than the upper part. Figure 2 shows an analogous situation for the microstructural evolution of prolate ellipsoids with aspect ratios 4:1:1.

Figure 3 exhibits the fully transformed microstructure on the planes $x_{1}=0.1, x_{1}=0.5$ and $x_{1}=0.9$, respectively. The growing ellipsoids have aspect ratios: (a) 1:1:1; (b) $1: 1: 1$; (c) $1: 1: 1$, (d) $8: 8: 1$, (e) $8: 8: 1$, (f) $8: 8: 1$, (g) $8: 1: 1$, (h) $8: 1: 1$ and (i) $8: 1: 1$. As expected, the grain size decreases as the nuclei intensity increases from $x_{1}=0.1$ to $x_{1}=0.9$ in all cases. Prolate ellipsoids displays anisotropic growth more clearly compared with oblate ellipsoids.

\subsection{Volume fraction transformed against time}

For oblate ellipsoids, Figure 4a shows the mean volume density, $V_{V}(t, x)$ as a function of normalized time (See section 2.). Figure $4 \mathrm{~b}$ shows the volume fraction, $\mathbf{V}_{\mathbf{v}}(\mathbf{t})$, as a function of normalized time. 


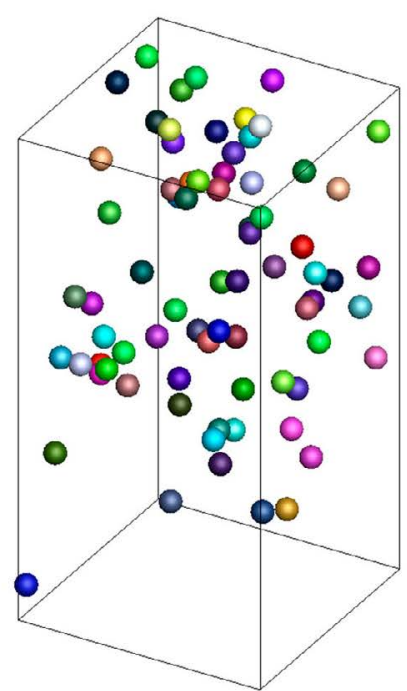

(a)

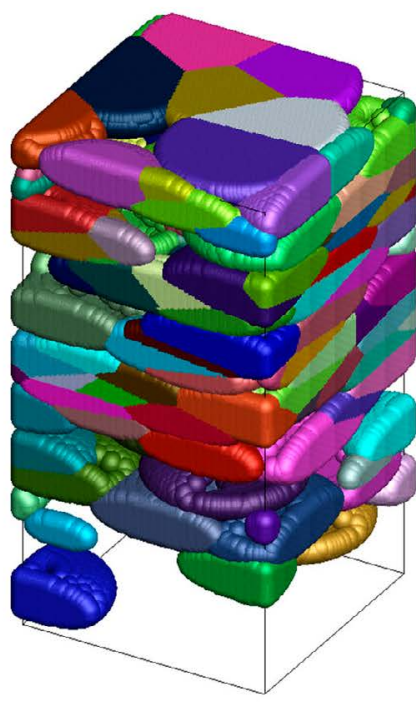

(b)

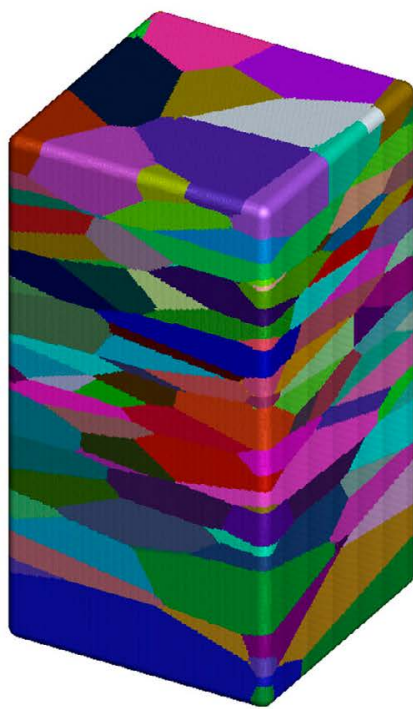

(c)

Figure 1. Computer simulated microstructures of the transformations inhomogeneous nucleated with grains that grow as oblate spheroids with aspect ratios 4:4:1: (a) $V_{V}=0$ (b) $V_{V}=0.3$, (c) $V_{V}=1$

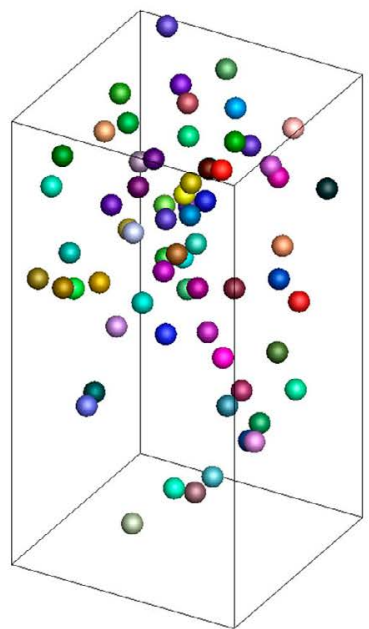

(a)

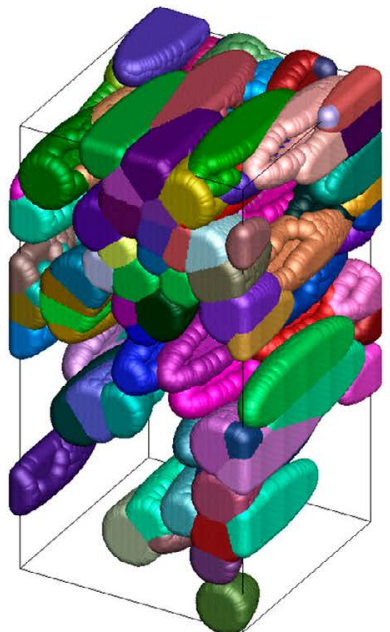

(b)

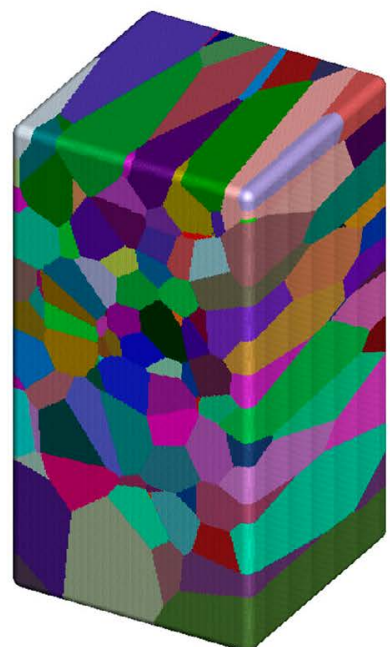

(c)

Figure 2. Computer simulated microstructures of the transformations inhomogeneous nucleated with grains that grow as prolate spheroids with aspect ratios 4:1:1: (a) $V_{V}=0$ (b) $V_{V}=0.3$, (c) $V_{V}=1$.

For prolate ellipsoids, Figure 5a exhibits the mean volume density, $V_{V}(t, x)$ as a function of normalized time. Figure $5 \mathrm{~b}$ shows the volume fraction, $\mathbf{V}_{\mathbf{v}}(\mathbf{t})$, as a function of normalized time.

We can compare the present work with previous work ${ }^{15}$ that used cellular automata to simulate the transformation nucleated according to an inhomogeneous Poisson point process. In the previous work ${ }^{15}$, the growing regions were not spherical but equiaxed owing to the use of cellular automata. In that work, analytical theory and experiment were also in good agreement.
The reference plane for the transformation kinetics is the plane with $x_{1}=0.5$. On this plane, one has $\lambda(0.5)=300$. This means that the transformation curve associated with $x_{1}=0.5$ is the same curve that would be obtained if the 300 nuclei were located within $[0,1] \mathrm{x}[0,1] \mathrm{x}[0,1]$ according to an homogeneous Poisson point process. Any plane above the reference plane has a faster kinetics as more nuclei are present. By contrast, any plane below the reference plane has a slower kinetics owing to the smaller number of nuclei.

Thanks to the use of normalized time the kinetics of all ellipsoids on a given plane in Figures $4 \mathrm{a}$ and $5 \mathrm{a}$ fell on 


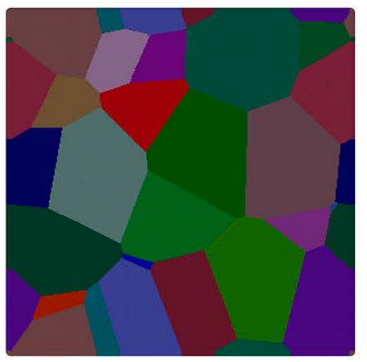

(a)

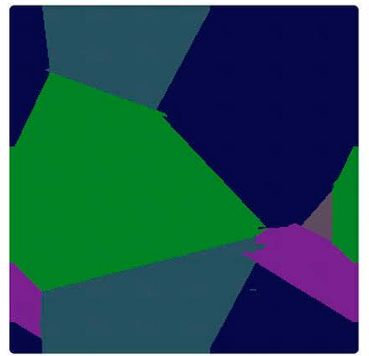

(d)

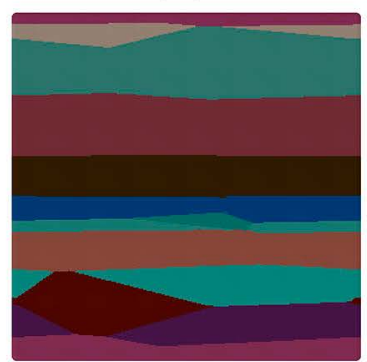

(g)

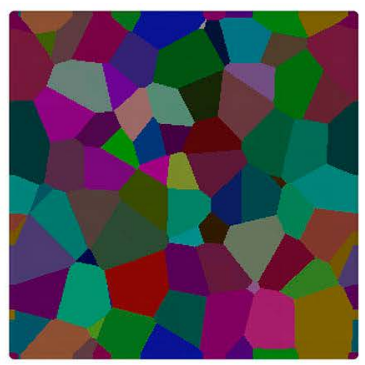

(b)

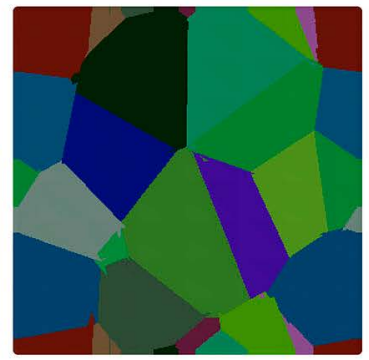

(e)

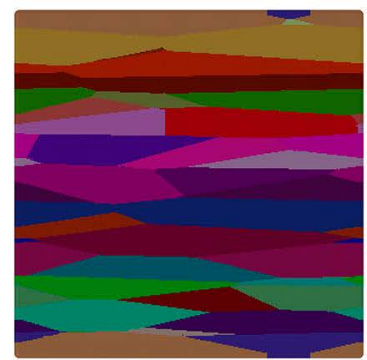

(h)

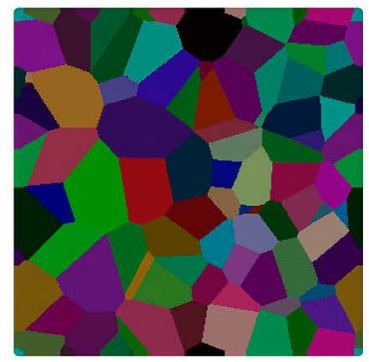

(c)

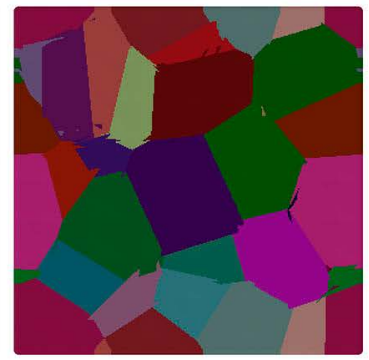

(f)

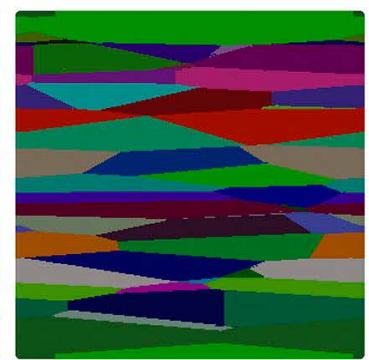

(i)

Figure 3. Fully transformed microstructure on $x_{1}$ sections with grains that grow with aspect ratios (a) $1: 1: 1$ on plane $x_{1}=0.1$; (b) $1: 1: 1$ on plane $x_{1}=0.5$; (c) $1: 1: 1$ on plane $x_{1}=0.9$; (d) $8: 8: 1$ on plane $x_{1}$ $=0.1$; (e) $8: 8: 1$ on plane $x_{1}=0.5$; (f) $8: 8: 1$ on plane $x_{1}=0.9$; (g) $8: 1: 1$ on plane $x_{1}=0.1$; (h) $8: 1: 1$ on plane $x_{1}=0.5$; and (i) $8: 1: 1$ on plane $x_{1}=0.9$;

the same line for a given value of $x_{1}$. This means that the kinetics of the simulation carried out here could be reduced to the kinetics of the spherical growth. But one must bear in mind that a critical condition for this to be possible is that $\lambda(x)$ must satisfy Eq. (9) for all values of $a_{i \cdot}$ Only when $\lambda(x)$ satisfies Eq. (9) one can obtain Eq. (10) formally similar to the equation derived for spherical growth by Rios and Villa $^{14}$. One can recall that, for spherical growth, as already said above, it is enough that $\lambda(x)$ is a harmonic function, that is, $\Delta \lambda(x)=0$.

Moreover, notice that, according to Rios and Villa ${ }^{14}$, if the nucleation takes place too close to $x_{1}=0$ or $x_{1}=1$ one must introduce correction factors. These correction factors are necessary to compensate for the fact that a region cannot grow beyond $x_{1}=0$ or $x_{1}=1$. As mentioned in the Methodology, there are no periodic boundary conditions on the top, $x_{1}=0$, and bottom, $x_{1}=1$, plane. Periodic boundary conditions were used only on the $x_{2}=0$ and $x_{2}=1$ and on he $x_{3}=0$ and $x_{3}=1$ planes. In the present case, transformations nucleated on $x_{1}=0.1$ or $x_{1}=0.9$ still gave good agreement with analytical theory without correction.

A significant difference from a transformation homogeneously nucleated is that the volume fraction transformed has to be calculated integrating over the total volume $[0,1] \times[0,1] \times[0,1]$, Eq. 14 . The volume fraction against time curves are shown in Figure $4 \mathrm{~b}$ and $5 \mathrm{~b}$. Likewise Figures $4 \mathrm{a}$ and $5 \mathrm{a}$, all curves can be normalized to fall on a single curve. One critical point is that the volume fraction curves showed in Figures $4 \mathrm{~b}$ and $5 \mathrm{~b}$ are different from the curve shown in Figures $4 a$ and $5 a$ for $x_{1}=0.5$. In other words, the volume fraction against time curves of Figures $4 \mathrm{~b}$ and $5 \mathrm{~b}$ do not coincide with the curve that would be obtained if the 300 nuclei were located within $[0,1] \times[0,1]$ $\mathrm{x}[0,1]$ according to an homogeneous Poisson point process. The volume fraction against time curves are significantly influenced by the inhomogeous nucleation. 


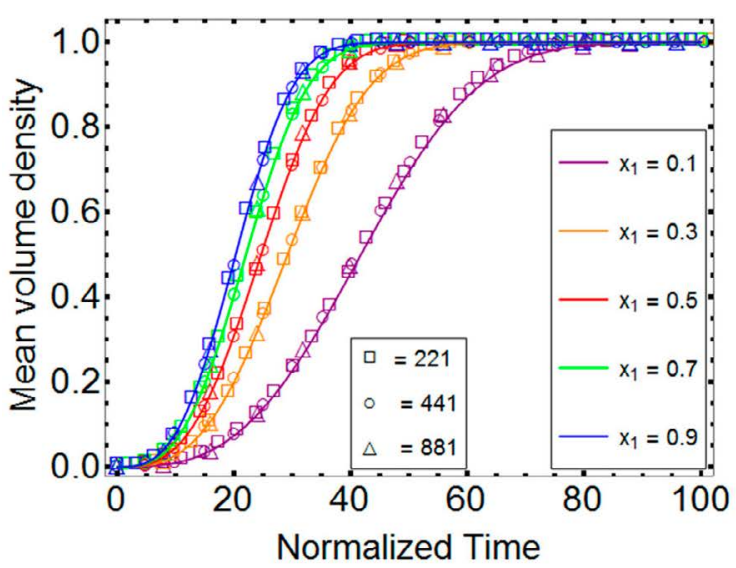

(a)

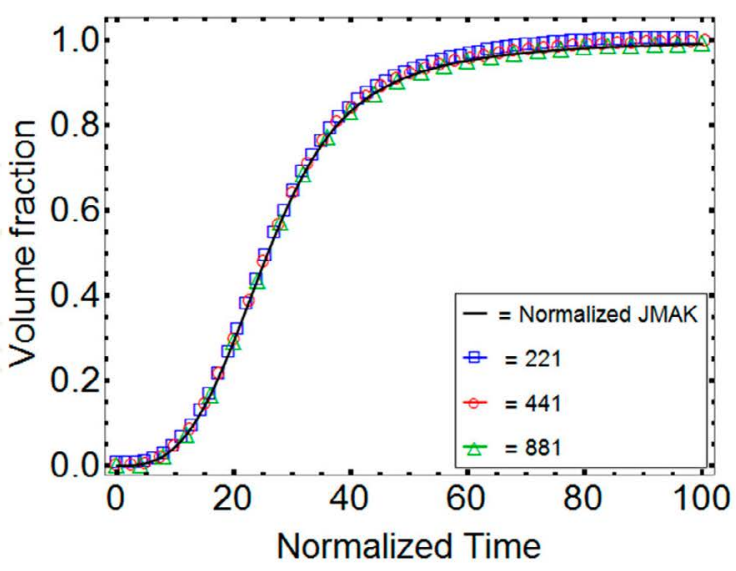

(b)

Figure 4. Transformation kinetics for oblate ellipsoids with aspect ratios: 2:2:1, 4:4:1, 8:8:1. (a) Mean volume density as a function of normalized time; (b) Volume fraction as a function of normalized time.

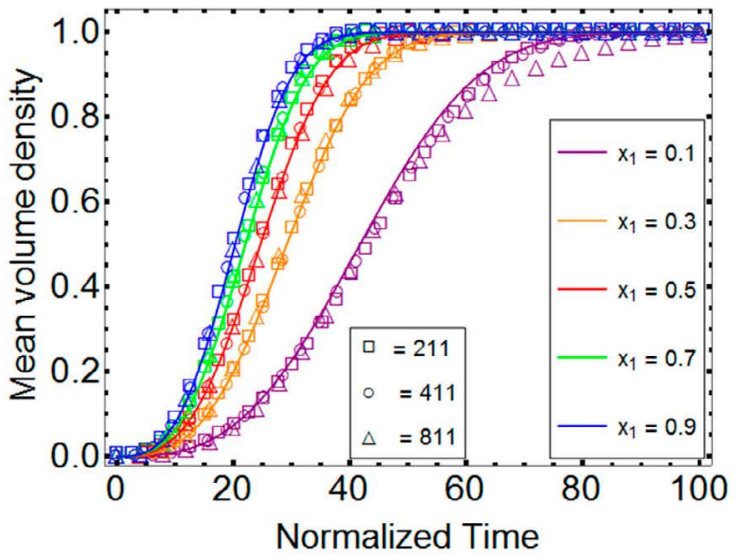

(a)

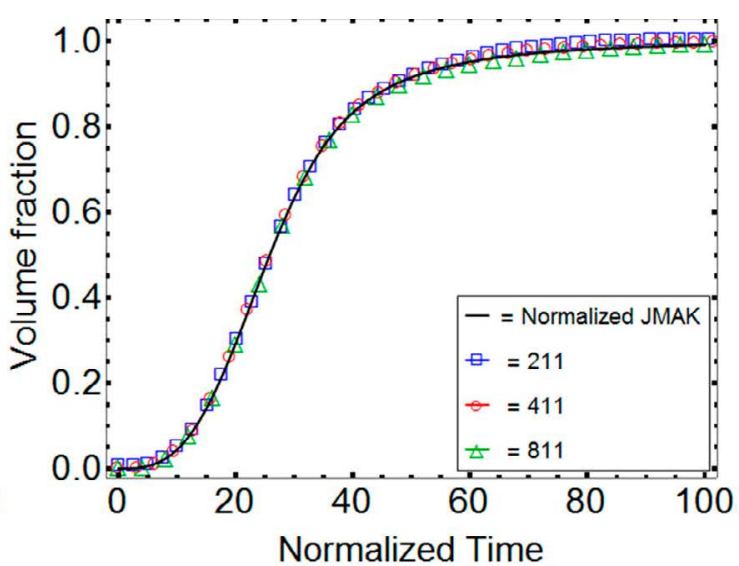

(b)

Figure 5. Transformation kinetics for prolate ellipsoids with aspect rations: 2:1:1, 4:1:1, 8:1:1. (a) Mean volume density as a function of normalized time; (b) Volume fraction as a function of normalized time.

\subsection{Contiguity}

Contiguity is defined as ${ }^{17}$

$$
C^{\beta \beta}=\frac{2 S_{v}^{\alpha \beta}}{2 S_{v}^{\alpha \beta}+S_{v}^{\beta \beta}}
$$

In Eq. (15) is the parent phase and $\beta$ is the new phase. $S_{v}^{\alpha \beta}$ is the interface area per unit of volume between the new phase and the parent phase. $S_{v}^{\beta \beta}$ is the interface area per unit of volume between the new phase. Both interfacial areas: $S_{v}^{\alpha \beta}$ and $S_{v}^{\beta \beta}$ were obtained from the microstructures generated by computer simulation.

This parameter allows quantifying the impingement of the grains within the 3D matrix. According to Vandermeer ${ }^{17}$, the contiguity detects deviations from nucleation randomness. For example, if nuclei form clusters or are arranged periodically, the contiguity will be significantly different ${ }^{17}$.
Figure 6 shows a plot of the contiguity measured over the total volume $[0,1] \mathrm{x}[0,1] \mathrm{x}[0,1]$ as a function of the volume fraction transformed. Figure 6 shows for nucleation according to an inhomogeneous Poisson point process the contiguity of spheres, dashed line in Figure 6, and ellipsoids of different shapes, symbols on Figure 6, fall on the same line. A similar phenomenon takes place when spheres, solid line in Figure 6, and ellipsoids (not shown) nucleate according to a homogeneous Poisson point process ${ }^{9}$. Figure 6 demonstrates that the contiguity curve of a transformation nucleated according to an inhomogeneous Poisson point process lies above the contiguity curve of a transformation nucleated according to a homogeneous Poisson point process. The contiguity is considered a reliable parameter to determine the arrangement of grains in space relative to one another ${ }^{10}$. According to Vandermeer ${ }^{10}$, when the contiguity curve of a transformation lies below the line for homogeneous Poisson 


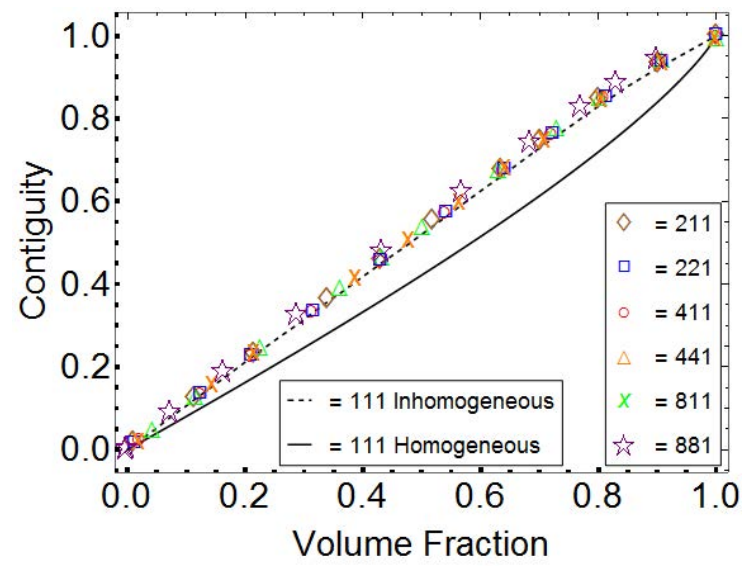

Figure 6. Contiguity against volume fraction. Solid line: the growth of spheres nucleated according to a homogeneous Poisson point process. Dashed line: the growth of spheres nucleated according to an inhomogeneous Poisson point process. The symbols correspond to ellipsoids nucleated according to an inhomogeneous Poisson point process

point process nucleation, the solid line in Figure 6, this indicates that the nuclei tend to a periodic arrangement. By contrast, when the contiguity curve of a transformation lies above the line for homogeneous Poisson point process nucleation, this indicates that the nuclei tend to cluster. Nuclei clusters have a broad meaning. Whenever there is a group of nuclei closer to one another than the average distance between nuclei, one has a nuclei cluster. For example, when nuclei form at the grain boundary of a polycrystal, they form a cluster, and their subsequent transformation has a contiguity curve above that of a homogeneous Poisson point process. In the present case, the inhomogeneous Poisson point process results in more nuclei located near than near $x_{1}=1$ than near $x_{1}=0$. Therefore, one might say that there is a clustering effect of nuclei at the upper part of the transformation volume. This is reflected in the fact that the contiguity curve for nucleation according to an inhomogeneous Poisson point process lies above the curve for nucleation according to a homogeneous Poisson point process. This behavior is coherent with Vandermeer ${ }^{10}$ observation. It is also coherent with computer simulation by Rios et al. ${ }^{18}$ that systematically changed nuclei location: from periodic to clustered. Rios et al. ${ }^{18}$ results were totally in agreement with Vandermeer's statement that was based on experimental observation.

\section{Conclusions}

- This paper further generalizes previous work of Rios and Villa on spherical growth ${ }^{14}$. The generalized equations, Eq. (10) and Eq. (11), apply to nucleation of ellipsoids according to an inhomogeneous Poisson point process.

- Spherical growth is a particular case of the more general equation derived here. However, the general equations, Eq. (10) and Eq. (11), manage to preserve the convenient form of the spherical growth equation.

- Computer simulation results showed excellent agreement with the theoretical results.

- Moreover, the computer simulation generates the microstructures obtained by nucleation and growth of oblate and prolate ellipsoids. The microstructures allow better visualization of the effect of the ellipsoid shape on the microstructure.

- Furthermore, computer simulation permits the measurement of the interfacial area between transformed and untransformed regions as well as the interfacial area between the transformed regions. The determination of these interfacial areas permits the determination of the contiguity. In the contiguity against volume fraction plot, data from the sphere and all ellipsoids fall on the same curve. The contiguity curve for nucleation according to an inhomogeneous Poisson point process falls above the contiguity curve for nucleation according to a homogeneous Poisson point process. This behavior indicates that nucleation according to an inhomogeneous Poisson point process introduced a nucleus clustering effect.

\section{Acknowledgments}

This study was financed in part by the Coordenação de Aperfeiçoamento de Pessoal de Nível Superior - Brasil (CAPES) - Finance Code 001. The authors are grateful to Conselho Nacional de Desenvolvimento Científico e Tecnológico, CNPQ and Fundação de Amparo à Pesquisa do Estado do Rio de Janeiro, FAPERJ, for the financial support.

\section{References}

1. Hansen N. Cold deformation microstructures. Materials Science and Technology. 1990;6(11):1039-1047.

2. Tanaka T. Controlled rolling of steel plate and strip. International Metal Reviews. 1981;26(1):185-212.

3. Oyama T, Watanabe C, Monzen R. Growth kinetics of ellipsoidal $\omega$-precipitates in a $\mathrm{Ti}-20 \mathrm{wt} \%$ Mo alloy under compressive stress. Journal of Materials Science. 2016;51(19):8880-8887.

4. Avrami M. Kinetics of Phase Change. I: General Theory. Journal of Chemical Physics. 1939;7(12):1103-1112.

5. Kolmogorov AN. On the Statistical Theory of Metal Crystallization. DAN SSSR: Seriya Matematicheskaya. 1937;1(1):335-360.

6. Johnson WA, Mehl RF. Reaction Kinetics in Processes of Nucleation and Growth. Transactions of A.I.M.E. 1939;135:416442 .

7. Shepilov MP, Baik DS. Computer simulation of crystallization kinetics for the model with simultaneous nucleation of randomlyoriented ellipsoidal crystals. Journal of Non-Crystalline Solids. 1994;171(2):141-156. 
8. Weinberg MC, Birnie DP III. Transformation kinetics for randomly oriented anisotropic particles. Journal of NonCrystalline Solids. 1995;189(1-2):161-166.

9. Godiksen RB, Rios PR, Vandermeer RA, Schmidt S, Jensen DJ. Three-dimensional geometric simulations of random anisotropic growth during transformation phenomena. Scripta Materialia. 2008;58(4):279-282.

10. Vandermeer RA, Masumura RA, Rath BB. Microstructural paths of shape-preserved nucleation and growth transformations. Acta Metallurgica et Materialia. 1991;39(3):383-389.

11. Chiu SN, Stoyan D, Kendall WS, Mecke J, eds. Stochastic Geometry and Its Applications. Hoboken: John Wiley \& Sons; 2013.

12. Lokovic KS, Bergmann RB, Bill A. Nonequilibrium grain size distribution with generalized growth and nucleation rates. Journal of Materials Research. 2013;28(11):1407-1412.

13. Lokovic KS, Bergmann RB, Bill A. The Grain Size Distribution in Crystallization Processes With Anisotropic Growth Rate. MRS Proceedings. 2010;1245:A16-07.
14. Rios PR, Villa E. Transformation kinetics for inhomogeneous nucleation. Acta Materialia. 2009;57(4):1199-1208.

15. Rios PR, Jardim D, Assis WLS, Salazar TC, Villa E. Inhomogeneous Poisson point process nucleation: comparison of analytical solution with cellular automata simulation. Materials Research. 2009;12(2):219-224.

16. Cahn JW. The Time Cone method for Nucleation and Growth Kinetics on a Finite Domain. MRS Proceedings. 1995;398:425.

17. Vandermeer RA. Microstructural descriptors and the effects of nuclei clustering on recrystallization path kinetics. Acta Materialia. 2005;53(5):1449-1457.

18. Rios PR, Pereira LO, Oliveira FF, Assis WLS, Castro JA. Impingement function for nucleation on non-random sites. Acta Materialia. 2007;55(13):4339-4348. 